\title{
Astromicin Sulfate
}

National Cancer Institute

\section{Source}

National Cancer Institute. Astromicin Sulfate. NCI Thesaurus. Code C76147.

The sulfate salt form of astromicin, an aminoglycoside antibiotic isolated from

Micromonospora olivasterospora with antibacterial activity. Astromicin contains an epipurpurosamine disaccharide backbone. 